\title{
Interpretation of triacylglycerol profiles of palm oil, palm kernel oil and their binary blends
}

\begin{abstract}
The effects of lipase-catalyzed interesterification (IE) on changes in the chemical composition of palm oil (PO), palm kernel oil (PKO) and their binary blends at 3:1, 1:1 and 1:3 (w/w) ratios, using both 1,3 specific Rhizomucor miehei, (Lipozyme ${ }^{\mathrm{TM}}$ ) and non-specific Pseudomonas sp. lipases were evaluated. IE of the native PO and PKO showed very distinct chemical composition changes. Catalysis of PO, using both lipases, caused synthesis of more medium and long chain triacylglycerols (TAG), with MMM/OLL, MMP, OOO and PPP (M, myristic acid; O, oleic acid; L, linoleic acid; P, palmitic acid) increasing in concentration. In contrast, IE of PKO resulted in the formation of more short and medium chain TAG, with $\mathrm{LaLaO}$ and $\mathrm{LaMO}$ (La, lauric acid; C, capric acid) experiencing noteworthy increments. Both Rhizomucor miehei and Pseudomonas sp. lipases showed high affinity in hydrolyzing PO fatty acids, resulting in high TAG losses and formation of high percentages of partial glycerides while these lipases were found to enhance the synthesis process in IE of PKO. Catalysis of the three binary blends caused similar TAG compositional changes where the synthesis process focussed on the medium chain TAG, while hydrolysis was observed in the short and long chain TAG that showed corresponding decreases. Catalysis of the three blends was influenced by the major fraction of these blends. Among these blends, PO: PKO at a 1:1 ratio exhibited the highest degree of IE. The diversity and quantity of available TAG are postulated to be the main causes of the different catalytic activities in these binary blends with Pseudomonas sp. lipase showing a higher degree and rate of IE than R. miehei.
\end{abstract}

Keyword: Binary blends, Fatty acid preference, Lipase-catalyzed interesterification, Palm oil, Palm kernel oil, Positional specificity, Pseudomonas sp., Rhizomucor miehei, Triacylglycerol composition changes 